\title{
Diophantine equations with a finite number of solutions: Craig Smoryński's theorem, Harvey Friedman's conjecture, and Minhyong Kim's guess
}

\author{
Apoloniusz Tyszka
}

\begin{abstract}
Matiyasevich's theorem states that there is no algorithm to decide whether or not a given Diophantine equation has a solution in non-negative integers. Smoryński's theorem states that the set of all Diophantine equations which have at most finitely many solutions in non-negative integers is not recursively enumerable. We prove: (1) Smoryński's theorem easily follows from Matiyasevich's theorem, (2) Hilbert's Tenth Problem for $\mathbb{Q}$ has a negative solution if and only if the set of all Diophantine equations with a finite number of rational solutions is not recursively enumerable.
\end{abstract}

Key words and phrases: Davis-Putnam-Robinson-Matiyasevich theorem, Diophantine equation which has at most finitely many solutions in non-negative integers, Hilbert's Tenth Problem, Hilbert's Tenth Problem for $\mathbb{Q}$, Matiyasevich's theorem, recursively enumerable set, Smoryński's theorem.

\section{Introduction}

The Davis-Putnam-Robinson-Matiyasevich theorem states that every recursively enumerable set $\mathcal{M} \subseteq \mathbb{N}$ has a Diophantine representation; that is

$$
a \in \mathcal{M} \Longleftrightarrow \exists x_{1}, \ldots, x_{m} \in \mathbb{N} W\left(a, x_{1}, \ldots, x_{m}\right)=0
$$

for some polynomial $W$ with integer coefficients, see [7]. The representation (R) is said to be infinite-fold if for every $a \in \mathcal{M}$ the equation $W\left(a, x_{1}, \ldots, x_{m}\right)=0$ has infinitely many solutions $\left(x_{1}, \ldots, x_{m}\right) \in \mathbb{N}^{m}$. A stronger version of the Davis-Putnam-Robinson-Matiyasevich theorem states that each recursively enumerable subset of $\mathbb{N}$ has an infinite-fold Diophantine representation with 9 variables, see [4], [6], [7, p. 163], and [9, p. 243].

Martin Davis' theorem states that the set of all Diophantine equations which have at most finitely many solutions in positive integers is not recursive, see [1]. Craig Smoryński's theorem states that the set of all Diophantine equations which have at most finitely many solutions in non-negative integers is not recursively enumerable, see [8, p. 104, Corollary 1] and [9, p. 240]. Yuri Matiyasevich's theorem states that there is no algorithm to decide whether or not a given Diophantine equation has a solution in non-negative integers ([7]). The same is true for solutions in integers and for solutions in positive integers ([7]).

Matiyasevich's theorem easily follows from the Davis-Putnam-Robinson-Matiyasevich theorem without the use of Smoryński's theorem ([7]). Similarly, the stronger version of the Davis-PutnamRobinson-Matiyasevich theorem implies that Matiyasevich's theorem holds for Diophantine equations which have at most $k$ variables, where $k \geqslant 9$, see [7]. In section 3, we show that Smoryński's theorem easily follows from Matiyasevich's theorem. In section 4, we show that Hilbert's Tenth Problem for $\mathbb{Q}$ has a negative solution if and only if the set of all Diophantine equations with a finite number of rational solutions is not recursively enumerable. 


\section{Basic lemmas}

Let $\mathcal{P}$ denote the set of prime numbers, and let

$$
\mathcal{P}=\left\{p_{1}, q_{1}, r_{1}, p_{2}, q_{2}, r_{2}, p_{3}, q_{3}, r_{3}, \ldots\right\},
$$

where $p_{1}<q_{1}<r_{1}<p_{2}<q_{2}<r_{2}<p_{3}<q_{3}<r_{3}<\ldots$.

Lemma 1. For a non-negative integer $x$, let $\prod_{i=1}^{\infty} p_{i}^{\alpha_{i}} \cdot q_{i}^{\beta_{i}} \cdot r_{i}^{\gamma_{i}}$ be the prime decomposition of $x+1$. For every positive integer $n$, the mapping which sends $x \in \mathbb{N}$ into

$$
\left((-1)^{\alpha_{1}} \cdot \frac{\beta_{1}}{\gamma_{1}+1}, \ldots,(-1)^{\alpha_{n}} \cdot \frac{\beta_{n}}{\gamma_{n}+1}\right) \in \mathbb{Q}^{n}
$$

is a computable surjection from $\mathbb{N}$ onto $\mathbb{Q}^{n}$.

Lemma 2. A Diophantine equation $D\left(x_{1}, \ldots, x_{p}\right)=0$ has no solutions in non-negative integers (alternatively, integers, positive integers, rationals) $x_{1}, \ldots, x_{p}$ if and only if the equation $D\left(x_{1}, \ldots, x_{p}\right)+0 \cdot x_{p+1}=0$ has at most finitely many solutions in non-negative integers (respectively, integers, positive integers, rationals) $x_{1}, \ldots, x_{p+1}$.

Proof. We present the proof for solutions in non-negative integers. Let $\mathcal{A}_{1}$ denote the following statement: A Diophantine equation $D\left(x_{1}, \ldots, x_{p}\right)=0$ has no solutions in non-negative integers $x_{1}, \ldots, x_{p}$. Let $\mathcal{A}_{2}$ denote the following statement: The equation $D\left(x_{1}, \ldots, x_{p}\right)+0 \cdot x_{p+1}=0$ has at most finitely many solutions in non-negative integers $x_{1}, \ldots, x_{p+1}$. We need to prove that

$$
\left(\mathcal{A}_{1} \Rightarrow \mathcal{A}_{2}\right) \wedge\left(\mathcal{A}_{2} \Rightarrow \mathcal{A}_{1}\right)
$$

We present the proof that $\mathcal{A}_{1}$ implies $\mathcal{A}_{2}$. The statement $\mathcal{A}_{1}$ implies that the set of all tuples $\left(x_{1}, \ldots, x_{p+1}\right) \in \mathbb{N}^{p+1}$ which satisfy $D\left(x_{1}, \ldots, x_{p}\right)+0 \cdot x_{p+1}=0$ is empty. The empty set is finite. We present the proof that $\mathcal{A}_{2}$ implies $\mathcal{A}_{1}$. Assume, on the contrary, that non-negative integers $a_{1}, \ldots, a_{p}$ satisfy $D\left(a_{1}, \ldots, a_{p}\right)=0$. Then,

$$
\forall x_{p+1} \in \mathbb{N} D\left(a_{1}, \ldots, a_{p}\right)+0 \cdot x_{p+1}=0
$$

Therefore, infinitely many tuples $\left(x_{1}, \ldots, x_{p+1}\right) \in \mathbb{N}^{p+1}$ solve the equation $D\left(x_{1}, \ldots, x_{p}\right)+0 \cdot x_{p+1}=0$, a contradiction. The proof for solutions in integers (positive integers, rationals) is analogous.

Lemma 3. If the set of all Diophantine equations which have at most finitely many solutions in non-negative integers (alternatively, integers, positive integers, rationals) is recursively enumerable, then there exists an algorithm which decides whether or not a given Diophantine equation has a solution in non-negative integers (respectively, integers, positive integers, rationals).

Proof. We present the proof for solutions in non-negative integers. Suppose that $\left\{\mathcal{S}_{i}=0\right\}_{i=0}^{\infty}$ is a computable sequence of all Diophantine equations which have at most finitely many solutions in non-negative integers. By Lemma 2, the execution of Flowchart 1 decides whether or not a Diophantine equation $D\left(x_{1}, \ldots, x_{p}\right)=0$ has a solution in non-negative integers. The flowchart algorithm uses a computable surjection $\varphi: \mathbb{N} \rightarrow \mathbb{N}^{p}$. 


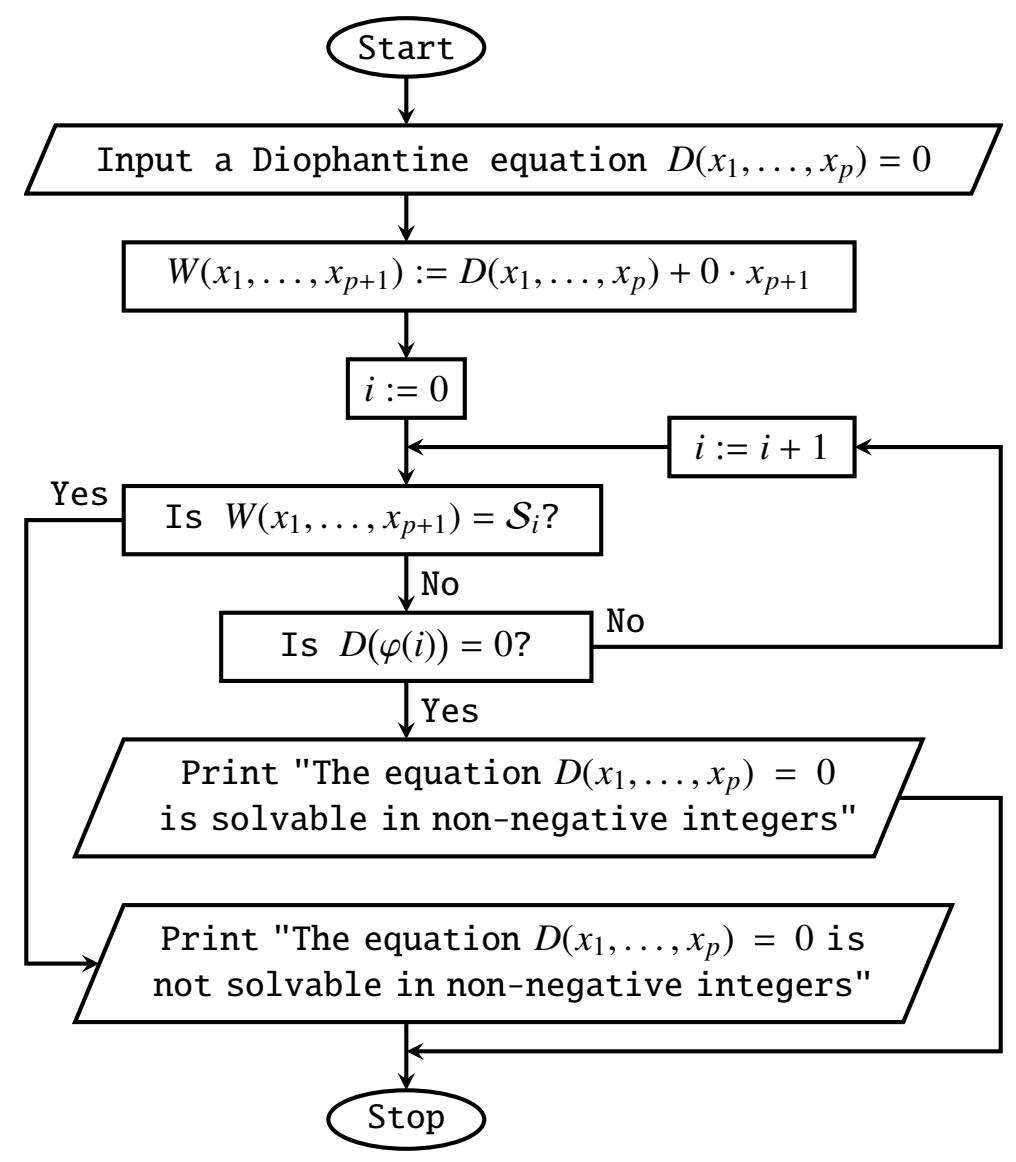

Flowchart 1

The flowchart algorithm always terminates because there exists a non-negative integer $i$ such that

$$
\left(D\left(x_{1}, \ldots, x_{p}\right)+0 \cdot x_{p+1}=\mathcal{S}_{i}\right) \vee(D(\varphi(i))=0)
$$

Indeed, for every Diophantine equation $D\left(x_{1}, \ldots, x_{p}\right)=0$, the flowchart algorithm finds a solution in non-negative integers, or finds the equation $D\left(x_{1}, \ldots, x_{p}\right)+0 \cdot x_{p+1}=0$ on the infinite list $\left[\mathcal{S}_{0}, \mathcal{S}_{1}, \mathcal{S}_{2}, \ldots\right]$ if the equation $D\left(x_{1}, \ldots, x_{p}\right)=0$ is not solvable in non-negative integers.

For solutions in integers, we choose a computable surjection $\varphi: \mathbb{N} \rightarrow \mathbb{Z}^{p}$, modify the definition of the sequence $\left\{\mathcal{S}_{i}=0\right\}_{i=0}^{\infty}$, and modify the two print instructions. For solutions in positive integers, we choose a computable surjection $\varphi: \mathbb{N} \rightarrow(\mathbb{N} \backslash\{0\})^{p}$, modify the definition of the sequence $\left\{\mathcal{S}_{i}=0\right\}_{i=0}^{\infty}$, and modify the two print instructions. For solutions in rationals, we apply Lemma 1 and choose a computable surjection $\varphi: \mathbb{N} \rightarrow \mathbb{Q}^{p}$, modify the definition of the sequence $\left\{\mathcal{S}_{i}=0\right\}_{i=0}^{\infty}$, and modify the two print instructions.

\section{The set of all Diophantine equations which have at most finitely many solutions in non-negative integers is not recursively enumerable}

Theorem 1. The set of all Diophantine equations which have at most finitely many solutions in non-negative integers (integers, positive integers) is not recursively enumerable.

Proof. It follows from Lemma 3 and Matiyasevich's theorem.

Let $\mathcal{E}$ denote the set of all Diophantine equations $D\left(x_{1}, \ldots, x_{p}\right)=0$ such that $p \in \mathbb{N} \backslash\{0\}$ and the polynomial $D\left(x_{1}, \ldots, x_{p}\right)$ truly depends on all the variables $x_{1}, \ldots, x_{p}$. The last phrase means that for every $i \in\{1, \ldots, p\}$ the polynomial $D\left(x_{1}, \ldots, x_{p}\right)$ involves a non-zero monomial which is divided by $x_{i}$, if $D\left(x_{1}, \ldots, x_{p}\right)$ is written as the sum of a minimal number of monomials. 
Lemma 4. A Diophantine equation $D\left(x_{1}, \ldots, x_{p}\right)=0$ has no solutions in non-negative integers $x_{1}, \ldots, x_{p}$ if and only if the equation $\left(2 x_{p+1}+1\right) \cdot D\left(x_{1}, \ldots, x_{p}\right)=0$ has at most finitely many solutions in non-negative integers $x_{1}, \ldots, x_{p+1}$.

Lemma 5. If a polynomial $D\left(x_{1}, \ldots, x_{p}\right) \in \mathbb{Z}\left[x_{1}, \ldots, x_{p}\right]$ truly depends on all the variables $x_{1}, \ldots, x_{p}$, then the polynomial $\left(2 x_{p+1}+1\right) \cdot D\left(x_{1}, \ldots, x_{p}\right)$ truly depends on all the variables $x_{1}, \ldots, x_{p+1}$.

Theorem 2. The equations which belong to $\mathcal{E}$ and which have at most finitely many solutions in non-negative integers form a set which is not recursively enumerable.

Proof. We reformulate Lemma 3 for Diophantine equations which belong to $\mathcal{E}$. The proof, which uses Lemmas $3 \sqrt{5}$, is analogous to the proof of Theorem 1 .

For a positive integer $k$, let $\operatorname{Dioph}(k)$ denote the set of all Diophantine equations which have at most $k$ variables and at most finitely many solutions in non-negative integers.

Theorem 3. For every integer $k \geqslant 9$, the set Dioph $(k)$ is not recursively enumerable.

Proof. Let $\left\{D_{j}=0\right\}_{j=0}^{\infty}$ be a computable sequence of all Diophantine equations which have at most $k$ variables. By the stronger version of the Davis-Putnam-Robinson-Matiyasevich theorem, there exists a polynomial $W\left(x, x_{1}, \ldots, x_{9}\right) \in \mathbb{Z}\left[x, x_{1}, \ldots, x_{9}\right]$ such that for every non-negative integer $j$, the equation $D_{j}=0$ is solvable in non-negative integers if and only if the equation $W\left(j, x_{1}, \ldots, x_{9}\right)=0$ has infinitely many solutions in non-negative integers $x_{1}, \ldots, x_{9}$. Equivalently, for every non-negative integer $j$, the equation $D_{j}=0$ has no solutions in non-negative integers if and only if the equation $W\left(j, x_{1}, \ldots, x_{9}\right)=0$ has at most finitely many solutions in non-negative integers $x_{1}, \ldots, x_{9}$. Suppose, on the contrary, that $\left\{\mathcal{G}_{i}=0\right\}_{i=0}^{\infty}$ is a computable sequence of all equations from $\operatorname{Dioph}(k)$. Then, the execution of Flowchart 2 decides whether or not a Diophantine equation $D\left(x_{1}, \ldots, x_{p}\right)=0$ (where $p \leqslant k$ ) has a solution in non-negative integers $x_{1}, \ldots, x_{p}$. The flowchart algorithm uses a computable surjection $\varphi: \mathbb{N} \rightarrow \mathbb{N}^{p}$. 


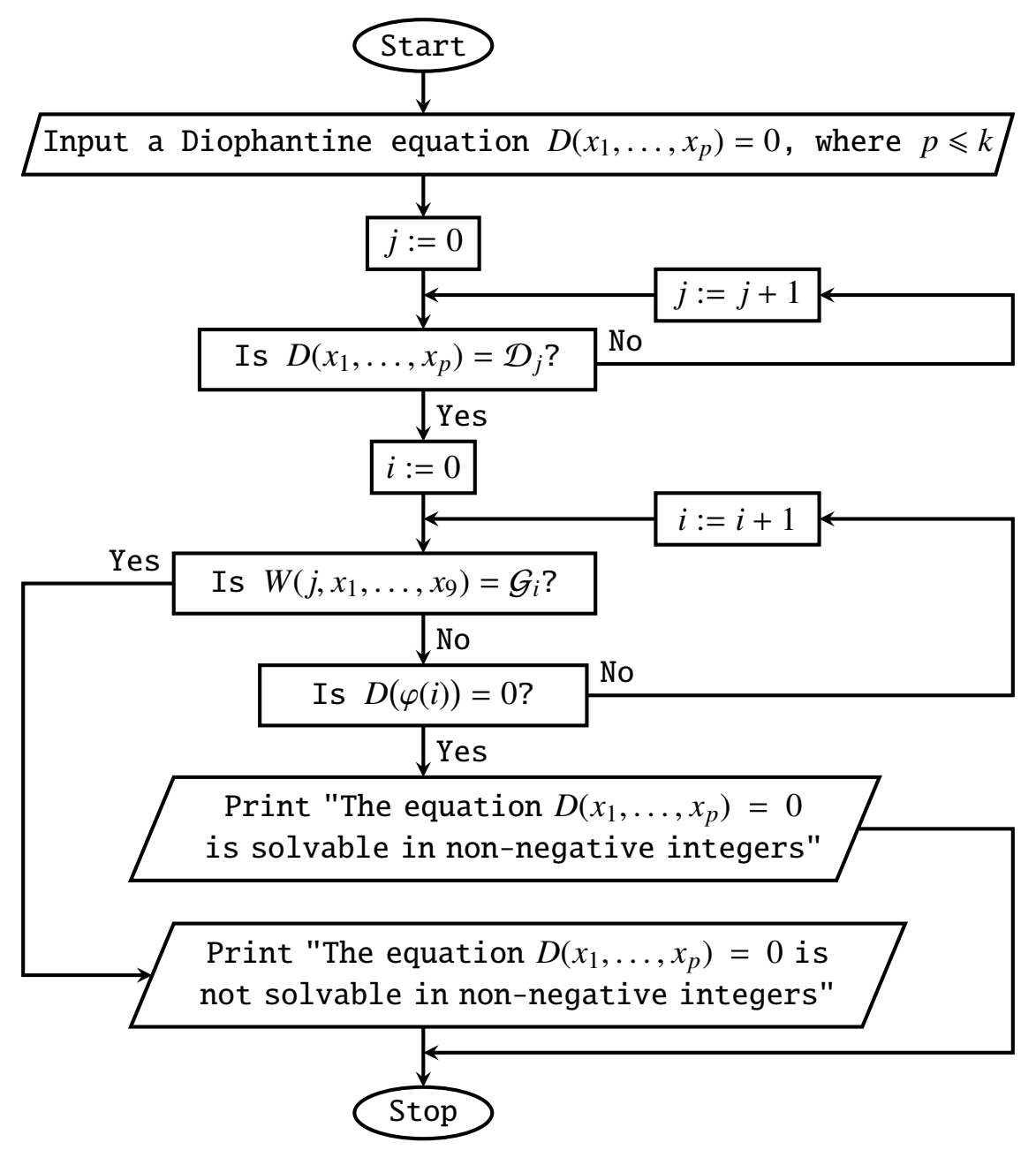

Flowchart 2

Thus we have a contradiction to Matiyasevich's theorem. The flowchart algorithm always terminates because there exist non-negative integers $i$ and $j$ such that

$$
\left(D\left(x_{1}, \ldots, x_{p}\right)=\mathcal{D}_{j}\right) \wedge\left(\left(W\left(j, x_{1}, \ldots, x_{9}\right)=\mathcal{G}_{i}\right) \vee(D(\varphi(i))=0)\right)
$$

\section{Hilbert's Tenth Problem for $\mathbb{Q}$}

Hilbert's Tenth Problem for $\mathbb{Q}$ remains unsolved, see [2] and [7]. For solutions in rationals, Lemma 3 claims that a negative solution to Hilbert's Tenth Problem for $\mathbb{Q}$ implies that the set of all Diophantine equations with a finite number of rational solutions is not recursively enumerable. We show the converse implication.

Theorem 4. A positive solution to Hilbert's Tenth Problem for $\mathbb{Q}$ implies that the set of all Diophantine equations with a finite number of rational solutions is recursively enumerable.

Proof. We assume a positive solution to Hilbert's Tenth Problem for $\mathbb{Q}$. The algorithm in Flowchart 3 halts if and only if a Diophantine equation $D\left(x_{1}, \ldots, x_{n}\right)=0$ has at most finitely many rational solutions. 


\section{Start}

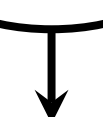

Input a Diophantine equation $D\left(x_{1}, \ldots, x_{n}\right)=0$

Fix a computable surjection $\zeta: \mathbb{N} \rightarrow \mathbb{Q}^{n}$

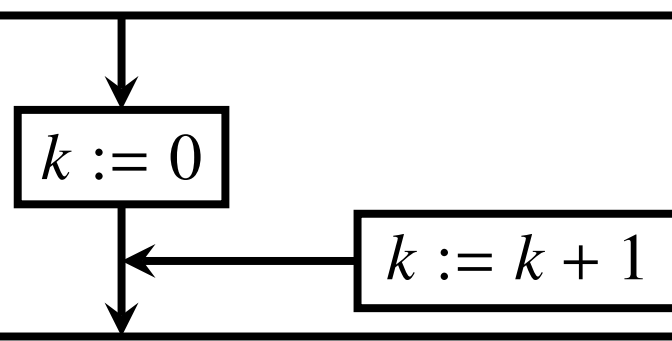

Does the equation $D^{2}\left(x_{1}, \ldots, x_{n}\right)+$ $\left(\left(y \cdot \prod_{\left(r_{1}, \ldots, r_{n}\right) \in\{\zeta(0), \ldots, \zeta(k)\}} \sum_{i=1}^{n}\left(x_{i}-r_{i}\right)^{2}\right)-1\right)^{2}=0$ is solvable in rationals $x_{1}, \ldots, x_{n}, y$ ?

No

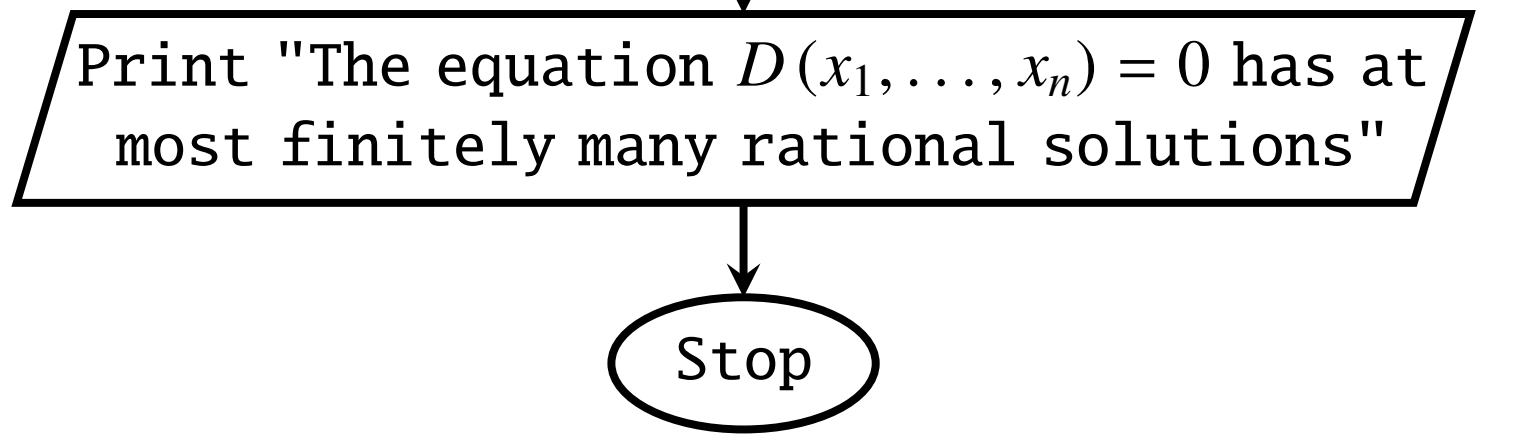

Flowchart 3

Harvey Friedman conjectures that the set of all Diophantine equations which have only finitely many rational solutions is not recursively enumerable, see [3].

Guess ([5, p. 16]). The question whether or not a given Diophantine equation has only finitely many rational solutions is decidable with an oracle that decides whether or not a given Diophantine equation has a rational solution.

Originally, Minhyong Kim formulated the Guess as follows: for rational solutions, the finiteness problem is decidable relative to the existence problem. 


\section{References}

[1] M. Davis, On the number of solutions of Diophantine equations, Proc. Amer. Math. Soc. 35 (1972), no. 2, 552-554, http://doi.org/10.1090/S0002-9939-1972-0304347-1.

[2] M. Davis, Representation theorems for recursively enumerable sets and a conjecture related to Poonen's large subring of $\mathbb{Q}$, J. Math. Sci. (N.Y.) 171 (2010), no. 6, 728-730, http://doi.org/ 10.1007/s10958-010-0176-7.

[3] H. Friedman, Complexity of statements, April 20, 1998, http://www.cs.nyu.edu/pipermail/ fom/1998-April/001843.html.

[4] J. P. Jones, Universal Diophantine equation, J. Symbolic Logic 47 (1982), no. 3, 549-571, http: //doi.org/10.2307/2273588.

[5] M. Kim, On relative computability for curves, Asia Pac. Math. Newsl. 3 (2013), no. 2, 16-20, http://www . asiapacific-mathnews.com/03/0302/0016_0020.pdf.

[6] Yu. Matiyasevich, Some purely mathematical results inspired by mathematical logic, in: Proceedings of Fifth International Congress on Logic, Methodology and Philosophy of Science, London, Ontario, 1975, Reidel, Dordrecht, 1977, 121Ú-127, http://doi.org/10.1007/978-94-010-1138-9_7.

[7] Yu. Matiyasevich, Hilbert's tenth problem, MIT Press, Cambridge, MA, 1993.

[8] C. Smoryński, A note on the number of zeros of polynomials and exponential polynomials, J. Symbolic Logic 42 (1977), no. 1, 99-106, http://doi.org/10.2307/2272324.

[9] C. Smoryński, Logical number theory, vol. I, Springer, Berlin, 1991.

Apoloniusz Tyszka

University of Agriculture

Faculty of Production and Power Engineering

Balicka 116B, 30-149 Kraków, Poland

E-mail: rttyszka@cyf-kr.edu.pl 